\title{
Prediction of risk of disease recurrence by genome-wide cDNA microarray analysis in patients with Philadelphia chromosome-positive acute lymphoblastic leukemia treated with imatinib-combined chemotherapy
}

\author{
HITOSHI ZEMBUTSU ${ }^{1}$, MASAMITSU YANADA ${ }^{2}$, ASAHI HISHIDA ${ }^{1}$, TOYOMASA KATAGIRI ${ }^{1}$, \\ TAKASHI TSURUO ${ }^{3}$, ISAMU SUGIURA ${ }^{4}$, JIN TAKEUCHI $^{5}$, NORIKO USUI ${ }^{6}$, \\ TOMOKI NAOE $^{2}$, YUSUKE NAKAMURA ${ }^{1}$ and RYUZO OHNO ${ }^{7}$
}

\begin{abstract}
${ }^{1}$ Laboratory of Molecular Medicine, Human Genome Center, Institute of Medical Science, The University of Tokyo, Tokyo 108-8639; ${ }^{2}$ Department of Hematology and Oncology, Nagoya University Graduate School of Medicine, Nagoya 466-8550; ${ }^{3}$ Cancer Chemotherapy Center, Cancer Research Institute, Tokyo 135-8550; ${ }^{4}$ Department of Hematology, Toyohashi Municipal Hospital, Toyohashi 441-8570; ${ }^{5}$ Department of Hematology and Rheumatology, Nihon University School of Medicine, Tokyo $173-8610 ;{ }^{6}$ Division of Hematology and Oncology, Jikei University School of Medicine, Tokyo 105-8461; ${ }^{7}$ Aichi Cancer Center, Nagoya 464-8681, Japan
\end{abstract}

Received March 19, 2007; Accepted May 4, 2007

\begin{abstract}
Philadelphia chromosome-positive acute lymphoblastic leukemia $(\mathrm{Ph}+\mathrm{ALL})$ reveals very poor prognosis due to high incidence of relapse when treated with standard chemotherapy. Although $>96 \%$ of patients with Ph+ALL achieved complete remission (CR) with imatinib-combined chemotherapy in a phase II clinical trial conducted by the Japan Adult Leukemia Study Group (JALSG), 26\% of them experienced hematological relapse in a short time after achievement of CR. In this study, to establish a prediction system for risk of relapse, we analyzed gene expression profiles of 23 bone marrow samples from patients with Ph+ALL using cDNA microarray consisting of 27,648 cDNA sequences. Using the 19 randomly-selected test cases, we identified 16 genes that were expressed significantly
\end{abstract}

Correspondence to: Dr Hitoshi Zembutsu, Laboratory of Molecular Medicine, Human Genome Center, Institute of Medical Science, The University of Tokyo, 4-6-1 Shirokanedai, Minato-ku, Tokyo 108-8639, Japan

E-mail: zembutsh@ims.u-tokyo.ac.jp

Abbreviations: ALL, acute lymphoblastic leukemia; $\mathrm{Ph}+$, Philadelphia chromosome-positive; CPM, cyclophosphamide; DNR, daunorubicin; VCR, vincristine; PSL, predonisolone; MTX, methotrexate; Ara-C, cytarabine; Dex, dexamethasone; mPSL, methylprednisolone; JALSG, Japan Adult Leukemia Study Group; $\mathrm{CR}$, complete remission; EFS, event-free survival

Key words: imatinib, BCR-Abl, acute lymphoblastic leukemia, prediction, microarray, recurrence differently between patients with $(n=8)$ and without $(n=11)$ continuous response; from the list of 16 genes, we selected the 6 'predictive' genes and constructed a numerical scoring system by which the two groups were clearly separated, with positive scores for the former and the negative scores for the latter. Scoring of 4 cases that were reserved from the original 23 cases predicted correctly their clinical responses. In addition, three cases whose BCR-Abl transcript levels failed to reduce sufficiently after induction therapy, also revealed negative scores. We also developed a quantitative reverse transcription-PCR-based prediction system that could be feasible for routine clinical use. Our results suggest that achievement of continuous response with imatinib-combined chemotherapy can be predicted by expression patterns in this set of genes, leading to achievement of 'personalized therapy' for treatment of this disease.

\section{Introduction}

Philadelphia chromosome-positive acute lymphoblastic leukemia $(\mathrm{Ph}+\mathrm{ALL})$ is characterized by a reciprocal translocation between chromosome 9 and chromosome $22[\mathrm{t}(9 ; 22)$ (q34;q11)], leading to the formation of the BCR-Abl fusion protein. This translocation is observed in about $3-5 \%$ of pediatric ALL and 20-30\% of adult ALL, and is usually associated with very poor prognosis $(1,2)$. Recently-developed chemotherapy regimens for adult Ph-negative ALL are very effective; $70-90 \%$ of the patients reach complete response (CR) and 30-50\% of them achieve long-term survival (3-7). Although patients with $\mathrm{Ph}+\mathrm{ALL}$ reveal almost similar CR rates ranging $60-80 \%$ with the same chemotherapy regimens, most of them experience relapse and die of the disease; the 5 -year survival rates are $<10 \%$ for adults $(8-11)$. However, their long-term event-free survival (EFS) rate is expected to 
A)

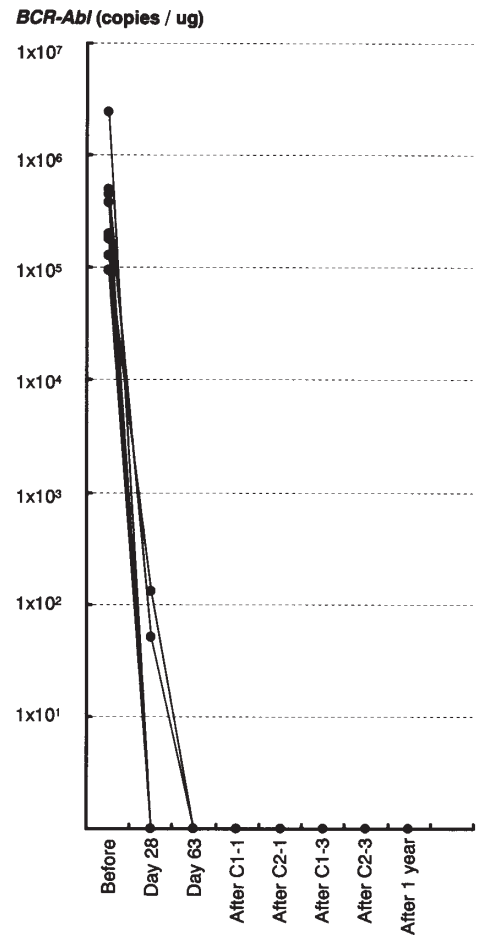

B)

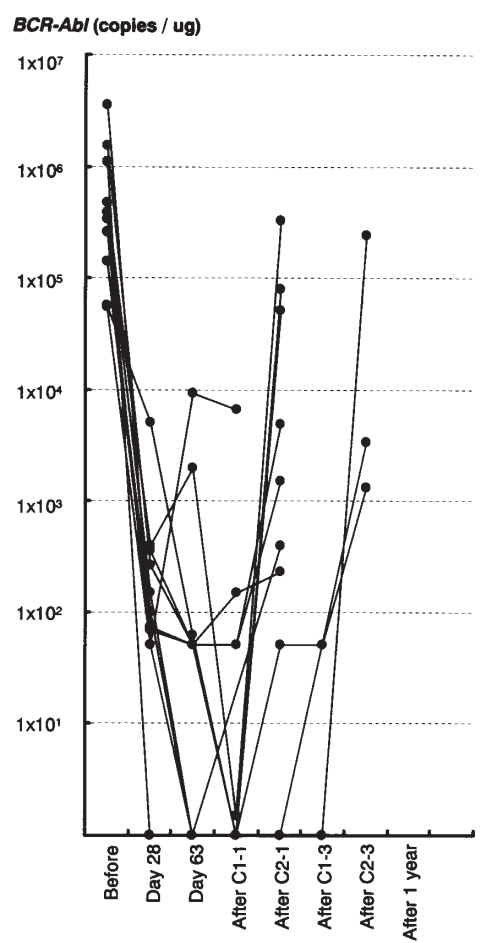

Figure 1. Molecular responses in 19 patients with Ph+ALL treated with imatinib-combined chemotherapy. Each line represents the BCR-Abl levels for patients with (A; group A) and without (B; group B) continuous response. The copy numbers of $B C R$ - $A b l$ in y-axis were measured by quantitative real-time RT-PCR. The day numbers in $\mathrm{X}$-axis indicate days after initiation of the remission induction therapy. C1-1 indicates the first cycle of consolidation 1 (C1), C2-1 the first cycle of consolidation 2 (C2), C1-3 the third cycle of $\mathrm{C} 1$ and $\mathrm{C} 2-3$ the third cycle of $\mathrm{C} 2$.

be as high as $40 \%$ if they undergo allogeneic hematopoietic stem-cell transplantation (HSCT) during first CR (12-18).

The Abl-selective tyrosine kinase inhibitor imatinib, which binds specifically to the nucleotide-binding pocket in the catalytic domain of BCR-Abl (19), has been developed, and is becoming the standard therapeutic drug for patients with chronic myelogenous leukemia (CML) (20-22). For Ph+ALL harboring the same chromosome abnormality, imatinib alone was able to induce good response in a substantial proportion of $\mathrm{Ph}+\mathrm{ALL}$ patients, but the response was not durable $(17,23)$. Hence, several groups are currently exploring a combination of imatinib and chemotherapy, and encouraging results have been published $(20,24-27)$. The Japan Adult Leukemia Study Group (JALSG) conducted a phase II clinical trial of imatinib-combined chemotherapy for adult patients with newly diagnosed Ph+ALL (27), and reported that CR was achieved in 77 of 80 patients $(96.2 \%)$. Nevertheless, 20 of the $77 \mathrm{CR}$ patients $(26 \%)$ experienced relapse within a relatively short follow-up period.

Generally, allogeneic HSCT has been considered the only curative form of treatment available for adults with this disease. However, it is associated with high risk of treatment-related morbidity and mortality. In fact, among 23 deaths reported in the article (27), 13 (57\%) were due to transplantation-related complications (27). Additionally, second generation tyrosine kinase inhibitors with more potent in vitro and in vivo activities are under development, and some of them have shown significant clinical efficacy in imatinib-resistant Ph-positive leukemias $(28,29)$. Taken together, there is a growing importance of discriminating patients who should be treated with imatinib-combined chemotherapy and those who should be offered HSCT or second generation tyrosine kinase inhibitors at the early stage of their disease before experiencing recurrence.

In this study, we analyzed the gene expression profile of $26 \mathrm{Ph}+\mathrm{ALL}$ cases using cDNA microarray representing 27,648 genes, and established a system to predict a risk of disease recurrence during imatinib-combined therapy. We report identification of 6 genes that showed significantly different levels of expression between patients with or without recurrence within 4 months after achievement of CR. We suggest that such information may lead ultimately to our goal of 'personalized therapy'.

\section{Materials and methods}

Patients and treatments. The JALSG Ph+ALL202 trial was open for patients with newly diagnosed Ph+ALL aged 15-64 years. Eligibility criteria included adequate functioning of the liver, kidneys, and heart and an Eastern Cooperative Oncology Group performance status between 0 and 3 . Written informed consent was obtained from all patients before registration $(25,27)$.

The design of the trial was previously described in detail (27). RNAs were isolated from bone marrow cells collected before start of treatment, and were subjected to the multiplex reverse-transcription-PCR (RT-PCR) test and this microarray analysis. All patients positive for $B C R-A b l$ were treated with imatinib-combined chemotherapy. For remission induction therapy, imatinib was administered from day 8 to day 63 in 
combination with cyclophosphamide (CPM), daunorubicin (DNR), vincristine (VCR), and predonisolone (PSL). Consolidation therapy consisted of an odd course (C1) comprising high-dose methotrexate (MTX) and high-dose cytarabine (Ara-C), and an even course (C2) with singleagent imatinib for 28 days. $\mathrm{C} 1$ and $\mathrm{C} 2$ were alternated for four cycles each. After the completion of the consolidation therapy, patients received maintenance therapy consisting of VCR, PSL, and imatinib up to 2 years from the date they had attained CR. The daily dose of imatinib used in this study was $600 \mathrm{mg}$.

Of patients registered in the JALSG trial, 26 patients who met all of the following criteria were eligible for the present study: i) approval of the microarray study by the institutional ethics committee; ii) samples for which informed consent was obtained for this analysis; iii) HSCT was not performed during the remission induction or consolidation therapy; iv) the proportion of blast cells exceeded $70 \%$; v) patients who survived longer than 2 months after the initiation of this treatment. According to the responses to the imatinib-combined chemotherapy, we categorized patients into 2 groups: patients whose $B C R-A b l$ level in bone marrow became undetectable during the remission induction therapy and did not increase throughout the observation period (group A), and patients whose $B C R$ - $A b l$ level in bone marrow once dropped below the threshold ( 50 copies/ $\mu \mathrm{g}$ total RNA) by quantitative realtime RT-PCR analysis, but increased within 4 months after achievement of CR (group B) as shown in Fig. 1.

RNA extraction and T7-based RNA amplification. Total RNAs from bone marrow cells of each Ph+ALL patient were extracted using QIAamp ${ }^{\circledR}$ RNA Blood Mini Kit (Qiagen, Valencia, CA), according to the manufacturer's instructions. To guarantee the quality of RNAs, total RNAs extracted from each sample were electrophoresed on a denaturing agarose gel, and the quality was confirmed by the presence of rRNA bands. Extracted total RNA samples were treated with DNase I (Nippon Gene, Tokyo, Japan), and T7-based RNA amplification was then carried out as described previously (30). After two rounds of RNA amplification, we obtained 40-350 mg of amplified RNA for each case. As a control, a mixture of total RNA extracted from mononuclear cells of 11 healthy volunteers was used. RNAs amplified by this method accurately reflected the proportions in the original RNA source, as we had confirmed earlier by semiquantitative RT-PCR experiments (31), in which data from the microarrays were consistent with the results obtained by RT-PCR.

cDNA microarray. To obtain cDNAs for spotting on the glass slides, we performed RT-PCR amplification for each gene, as described previously (31). The PCR products were spotted on Alice glass slides ${ }^{\circledR}$ (GE Healthcare, Amersham Biosciences, Buckinghamshire, UK) with a high-density Microarray Spotter Lucidea (GE Healthcare); 9,216 genes were spotted in duplicate on a single slide. We prepared three different sets of slides (a total of 27,648 gene spots), on each of which the same 52 housekeeping genes and 2 negative control genes were spotted as well. The cDNA probes were prepared from amplified RNA using the method described previously (30). For hybridization experiments,
$2.5 \mathrm{mg}$ of amplified RNAs from each patient and from the control were reversely transcribed in the presence of Cy5dCTP and Cy3-dCTP (GE Healthcare), respectively. Hybridization, washing, and detection of signals were carried out as described previously (30).

Quantification of signals. We quantified the signal intensities of $\mathrm{Cy} 3$ and Cy5 from the 27,648 spots and analyzed the signals by substituting backgrounds, using ArrayVision software (Imaging Research, Inc., St. Catharines, Canada). Subsequently, the fluorescence intensities of Cy5 (Ph+ALL) and $\mathrm{Cy} 3$ (control) for each target spot were adjusted so that the mean $\mathrm{Cy} 5 / \mathrm{Cy} 3$ ratio of the 52 house-keeping genes became one. Because the data with low signal intensities are less reliable, we defined a cut-off value for the data on each slide as described previously (32) and excluded genes from further analysis when both Cy3 and Cy5 signal-intensities were lower than the cut-off value (32).

For genes in which either or both of the signal-intensities were above the cut-off value, we calculated the ratio of $\mathrm{Cy} 5 / \mathrm{Cy} 3$ signals as a relative expression ratio using the raw data of each sample. However, if either of the Cy3 or Cy5 signal intensity was lower than the cut-off value, the $\mathrm{Cy} 5 / \mathrm{Cy} 3$ ratio might be calculated to an extremely high or low and lead to make a significant false-influence for the further analysis. Hence, when either Cy3 or Cy5 signal intensity was less than the cut-off value, we adjusted the lower one to be half of each cut-off value plus the signal intensities, and then calculated the $\mathrm{Cy} 5 / \mathrm{Cy} 3$ ratios.

Hierarchical clustering analysis. We used web-available software ('Cluster' and 'TreeView') written by M. Eisen (http://genome-www5.stanford.edu/MicroArray/SMD/ restech.html) to create a graphic representation of the microarray data and to create a dendrogram of hierarchical clustering. Before the clustering algorithm was applied, the fluorescence ratio for each spot was first log-transformed and then the data for each sample were median-centered to remove experimental biases.

Extraction of genes for predicting the disease recurrence. We applied a random permutation test to identify genes that were expressed at a significantly different level in leukemic cells between group A and B. Mean $(\mu)$ and standard deviation ( $\delta$ ) were calculated from the log transformed relative expression ratios of each gene in patients with and without continuous response. A discrimination score (DS) for each gene was defined as follows:

$$
\mathrm{DS}=\left(\mu_{\mathrm{A}}-\mu_{\mathrm{B}}\right) /\left(\delta_{\mathrm{A}}-\delta_{\mathrm{B}}\right)
$$

We carried out permutation tests to estimate the ability of individual genes to distinguish the two groups; samples were randomly permutated between the two groups 10,000 times. Because the DS data set of each gene showed a normal distribution, we calculated a P-value for the user-defined grouping (33). For the initial analysis, we applied the expression data for original 19 cases consisting of 8 in group A and 11 in group B. As the next step, we randomly excluded one case from each group and made 10 different 
combinations consisting of 7 in group A and 10 in group B. We performed additional 10 permutation tests using the 10 combinations of the two groups.

Calculation of prediction score. We calculated a prediction score according to the procedure described previously (33). Each gene $\left(g_{\mathrm{i}}\right)$ votes for either case in group A or group B depending on whether the expression level $\left(\mathrm{x}_{\mathrm{i}}\right)$ in the sample is closer to the mean expression level of group A or group B in reference samples. The magnitude of the vote $\left(\mathrm{V}_{\mathrm{i}}\right)$ reflects the deviation of the expression level in the sample from the average of the two classes:

$$
\mathrm{V}_{\mathrm{i}}=\mathrm{I} \mathrm{x}_{\mathrm{i}}-\left(\mu_{\mathrm{A}}+\mu_{\mathrm{B}}\right) / 2 \mathrm{I}
$$

We summed the votes to obtain total votes for the group A $\left(\mathrm{V}_{\mathrm{A}}\right)$ and group $\mathrm{B}\left(\mathrm{V}_{\mathrm{B}}\right)$, and calculated PS values as follows:

$$
\mathrm{PS}=\left[\left(\mathrm{V}_{\mathrm{A}}-\mathrm{V}_{\mathrm{B}}\right) /\left(\mathrm{V}_{\mathrm{A}}+\mathrm{V}_{\mathrm{B}}\right)\right] \times 100
$$

reflecting the margin of victory in the direction of either patient with or without continuous response. PS values range from -100 to 100; a higher absolute value of PS reflects a stronger prediction.

Evaluation of the classification and leave-one-out method. We calculated the classification score (CS) using prediction scores of patients with $\left(\mathrm{PS}_{\mathrm{A}}\right)$ and without $\left(\mathrm{PS}_{\mathrm{B}}\right)$ continuous responses in each gene set, as follows:

$$
\mathrm{CS}=\left[\mu\left(\mathrm{PS}_{\mathrm{A}}\right)-\mu\left(\mathrm{PS}_{\mathrm{B}}\right)\right] /\left[\delta\left(\mathrm{PS}_{\mathrm{A}}\right)+\delta\left(\mathrm{PS}_{\mathrm{B}}\right)\right]
$$

A larger value of CS indicates better separation of the two groups by the predictive-scoring system. For the leave-oneout test, one sample is withheld, the permutation P-value and mean expression levels are calculated using remaining samples, and the class of the withheld sample is subsequently evaluated by calculating its prediction score. We repeated this procedure for each of the 19 samples.

Quantitative reverse transcription-PCR. Aliquots of the same aRNA hybridized to the microarray slides from individual samples and from a mixture of pooled mRNA from peripheral blood of 11 healthy volunteers were reversely transcribed using oligo(dT) ${ }_{12-18}$ primer and SuperScript II reverse transcriptase (Invitrogen, Carlsbad, CA). Quantitative-RT-PCR were carried out using SYBR Premix Ex Taq (Takara, Ohtsu, Japan) on an ABI PRISM 7700 Sequence Detection system (Applied Biosystems, Foster, CA) as described previously (34). The sequences of primers used in the real-time PCR are shown in Table II. To normalize the expression of each gene, we selected as internal controls PGK1, ACTB, CCT6A and SFRS4 from among the 52 housekeeping genes because they showed the smallest $\mathrm{Cy} 5 / \mathrm{Cy} 3$ fluctuations in our microarray data. Because normalization to these four endogenous control genes (PGK1, ACTB, CCT6A and SFRS4) led to similar conclusions (data not shown), we subsequently recorded only the data normalized according to levels of PGK1 expression. For generation of standard curves we used a mixture of mRNAs derived from mononuclear fraction from 19 patients. Quantitative RT-PCR experiments were done in duplicate for all 6 'predictive' genes, and relative expression ratios of each sample were calculated. The normalized gene expression values were log-transformed (on a base 2 scale), in a manner similar to the transformation of microarray-based hybridization data.

\section{Results}

Patients characteristics. Characteristics of the 26 patients are summarized in Table I. There were 14 males and 12 females with the median age of 45.0 years (range, 15-63 years). Seven patients were positive for major BCR-Abl and 19 for minor BCR-Abl. All patients received the same remission induction, consolidation and maintenance therapy as described in Materials and methods. CR was achieved in 25 of 26 patients (96\%). After a median follow-up of 26.7 months, relapse occurred in 12 patients. The probabilities of EFS and overall survival at 2 years were $48.7 \%$ (SE, 10.1\%), and $60.0 \%$ (SE, 10.0\%), respectively. As shown in Fig. 1, we categorized patients into two groups according to the responses to the imatinib-combined chemotherapy.

Identification of genes associated with disease recurrence. To extract genes that were differentially expressed between the two groups, we first analyzed initial 19 samples (8 in group A and 11 in group B) by comparing expression levels of 27,648 genes. We carried out a random permutation test to identify genes that showed significantly different expression levels between the two groups, and identified dozens of genes whose permutation $\mathrm{P}$-values were $<1 \times 10^{-3}$ and whose signal intensities were higher than the cut-off level in $>60 \%$ of samples in at least one group ( $\%$ presence $>60$ ). To further select the genes that more effectively distinguish the 2 groups, we performed 10 additional random permutation tests with 10 different combinations of 7 cases in group A and 10 cases in group B that were selected randomly, because the number of samples for this analysis was limited. As shown in Table III, $T N K 2, G L T S C R 2$ and $A P 2 B 1$ were selected by all of the 10 tests; of course, the genes with smaller P-values in the primary random permutation test had a tendency to be selected frequently through these 10 additional tests. The additional random permutation tests further defined the 16gene set, which were commonly selected by more than four additional permutation tests and isolated by the same criteria as described above $\left(\mathrm{p}<1 \times 10^{-3}, \%\right.$ presence $\left.>60\right)$.

Establishment of predictive scoring system for disease recurrence. Using the 16-gene set that seemed to effectively distinguish the two groups, we calculated the prediction score of each sample by the weighted-vote method (34). Then we rank-ordered these candidates on the basis of the number of selected times by 10 permutation tests (Table III) and calculated prediction scores by the leave-one-out test for cross-validation. For the leave-one-out test, we withheld one sample and calculated the permutation P-values and mean expression levels using the remaining samples to identify genes that were the most powerful for separating the two groups. 
Table I. Patient characteristics.

\begin{tabular}{|c|c|c|c|c|c|c|c|}
\hline Age & Gender & $\begin{array}{c}\text { Response to } \\
\text { imatinib combined } \\
\text { chemotherapy }^{\mathrm{a}}\end{array}$ & $\begin{array}{l}\text { WBC count } \\
\quad(\operatorname{per} \mu 1)\end{array}$ & $\begin{array}{c}\text { Bone marrow } \\
\text { blast }(\%)\end{array}$ & $\begin{array}{l}\text { FAB } \\
\text { type }\end{array}$ & $\begin{array}{l}\text { Type of } \\
\text { BCR-Ab }\end{array}$ & $\begin{array}{c}\text { Copy number of } \\
\text { BCR-Abl }^{\mathrm{b}}\end{array}$ \\
\hline
\end{tabular}

\begin{tabular}{|c|c|c|c|c|c|c|c|}
\hline 57 & Female & Group A (learning) & 3,520 & 72.3 & L1 & Minor & $4.40 \times 10^{5}$ \\
\hline 59 & Female & Group A (learning) & 19,000 & 92 & $\mathrm{~L} 2$ & Major & $9.30 \times 10^{4}$ \\
\hline 28 & Female & Group B (learning) & 19,800 & 94 & $\mathrm{~L} 2$ & Minor & $1.40 \times 10^{5}$ \\
\hline 20 & Female & Group B (learning) & 141,000 & 97.6 & $\mathrm{~L} 2$ & Minor & $1.50 \times 10^{6}$ \\
\hline 48 & Male & Group A (learning) & 103,000 & 95 & $\mathrm{~L} 2$ & Minor & $3.80 \times 10^{5}$ \\
\hline 40 & Male & Group B (learning) & 46,500 & 90 & $\mathrm{~L} 2$ & Minor & $3.40 \times 10^{5}$ \\
\hline 34 & Male & Group B (learning) & 176,000 & 98 & $\mathrm{~L} 2$ & Minor & $3.80 \times 10^{5}$ \\
\hline 62 & Female & Group A (learning) & 26,800 & 95.9 & $\mathrm{~L} 2$ & Minor & $1.30 \times 10^{5}$ \\
\hline 63 & Male & Group B (learning) & 231,000 & 96 & $\mathrm{~L} 2$ & Minor & $1.10 \times 10^{6}$ \\
\hline 57 & Male & Group B (learning) & 85,800 & 95 & $\mathrm{~L} 2$ & Minor & $1.40 \times 10^{5}$ \\
\hline 63 & Female & Group A (learning) & 18,100 & 91.1 & L1 & Minor & $1.80 \times 10^{5}$ \\
\hline 52 & Male & Group A (learning) & 87,300 & 91.9 & $\mathrm{~L} 2$ & Major & $2.00 \times 10^{5}$ \\
\hline 54 & Female & Group B (learning) & 13,700 & 96.2 & $\mathrm{~L} 2$ & Minor & $4.70 \times 10^{5}$ \\
\hline 15 & Male & Group B (learning) & 75,100 & 97.2 & $\mathrm{~L} 2$ & Minor & $5.60 \times 10^{4}$ \\
\hline 45 & Male & Group A (learning) & 3,300 & 98 & $\mathrm{~L} 2$ & Minor & $4.90 \times 10^{5}$ \\
\hline 45 & Male & Group B (learning) & 139,000 & 98 & $\mathrm{~L} 2$ & Minor & $5.50 \times 10^{4}$ \\
\hline 30 & Male & Group B (learning) & 7,500 & 96.4 & $\mathrm{~L} 2$ & Major & $3.50 \times 10^{6}$ \\
\hline 35 & Female & Group A (learning) & 91,000 & 88.9 & $\mathrm{~L} 2$ & Major & $2.40 \times 10^{6}$ \\
\hline 56 & Female & Group B (learning) & 23,400 & 94.6 & $\mathrm{~L} 2$ & Minor & $2.60 \times 10^{5}$ \\
\hline 33 & Male & Group A (test) & 5,800 & 96.4 & $\mathrm{~L} 2$ & Major & $7.20 \times 10^{6}$ \\
\hline 38 & Female & Group A (test) & 4,000 & 93 & L2 & Major & $3.90 \times 10^{6}$ \\
\hline 59 & Female & Group A (test) & 122,000 & 94 & $\mathrm{~L} 2$ & Minor & $2.90 \times 10^{5}$ \\
\hline 37 & Male & Group A (test) & 18,800 & 92 & L1 & Minor & $4.40 \times 10^{5}$ \\
\hline 52 & Male & Group C & 54,500 & 90 & $\mathrm{~L} 2$ & Minor & $7.60 \times 10^{5}$ \\
\hline 22 & Female & Group C & 114,000 & 94.8 & $\mathrm{~L} 2$ & Major & $1.20 \times 10^{6}$ \\
\hline 21 & Male & Group C & 814,000 & 98 & $\mathrm{~L} 2$ & Minor & $5.30 \times 10^{5}$ \\
\hline
\end{tabular}

${ }^{a}$ Group A, patients whose BCR-Abl level in bone marrow became undetectable during remission induction therapy and indicated no increase thereafter. Group B, patients whose BCR-Abl level in bone marrow dropped below the threshold (50 copies/ $\mu \mathrm{g}$ total RNA) but increased within 4 months after achievement of CR. Group C, patients whose BCR-Abl level in bone marrow failed to decrease sufficiently after induction therapy. Test, samples used for validation of the prediction scoring system. ${ }^{\text {bC }}$ opy numbers of BCR-Abl before initiaton of imatinib-combined chemotherapy were measured by quantitative real-time RT-PCR.

Table II. The primer sets.

\begin{tabular}{|c|c|c|c|}
\hline Accession no. & Symbol & Forward primer & Reverse primer \\
\hline \multicolumn{4}{|c|}{ Internal controls } \\
\hline NM_000291 & $P G K 1$ & 5'-CAAATGGAACACGGAGGATAAAG-3' & 5'-CACAGGAACTAAAAGGCAGGAAA-3' \\
\hline NM_001101 & $A C T B$ & 5'-CCCTGGAGAAGAGCTACGAG-3' & 5'-TGAAGGTAGTTTCGTGGATGC-3' \\
\hline AF385084 & ССТ6А & 5'-AGGACCAAATAAGCACACACTCAC-3' & 5'-CACACAGCCATCATCAATAGCA-3' \\
\hline L14076 & SFRS4 & 5'-GGAAGGTCGAGGAGAGAGTGAG-3' & 5'-CTGGAGCGTGATTCTGATGG-3' \\
\hline \multicolumn{4}{|c|}{ Predictive genes } \\
\hline BC044310 & TNK2 & 5'-TCTTCAGCTCCACAACCTAAGACA-3' & 5'-GGTGCTACCGCTGTTCAAATC-3' \\
\hline NM_015710 & GLTSCR2 & 5'-CTTGTTGCAGAAGGGTTGAGG-3' & 5'-AAGAAGCTCAGGCGGAAGG-3' \\
\hline NM_001282 & $A P 2 B 1$ & 5'-GCTTCCCTCTTGGCTCATTC-3' & 5'-GGTGTTTGCTTCTCCTGCTCTT-3' \\
\hline NM_013286 & $R B M 15 B$ & 5'-ACTGTCTGCTGTGGTTCTGTATTTT-3' & 5'-GGATGTCTTCGATTCTTTTAGCTTG-3' \\
\hline NM_024834 & Cloorf119 & 5'-ATTAGCAGTACCTTGGGCTGTTTT-3' & 5'-TGCACCACCTCCTCCACTT-3' \\
\hline AA196770 & $A L S 2 C R 4$ & 5'-TAGGCCAGGCATGGATCTTA-3' & 5'-CCTCCAAAGGTGAGCTGGTA-3' \\
\hline
\end{tabular}


Table III. The 16 discriminating genes.

\begin{tabular}{|c|c|c|c|c|c|}
\hline $\begin{array}{l}\text { Accession } \\
\text { no. }\end{array}$ & $\begin{array}{l}\text { No. of } \\
\text { selected } \\
\text { times }\end{array}$ & P-value ${ }^{b}$ & Symbol & Gene name & Group $^{c}$ \\
\hline
\end{tabular}

\begin{tabular}{|c|c|c|c|c|c|}
\hline ВC044310 & 10 & $1.93 \times 10^{-6}$ & $T N K 2$ & Tyrosine kinase, non-receptor, 2 & - \\
\hline NM_015710 & 10 & $1.31 \times 10^{-5}$ & GLTSCR2 & Glioma tumor suppressor candidate region gene 2 & - \\
\hline NM_001282 & 10 & $3.11 \times 10^{-5}$ & $A P 2 B 1$ & Adaptor-related protein complex $2, \beta 1$ subunit & - \\
\hline NM_013286 & 9 & $1.46 \times 10^{-8}$ & $R B M 15 B$ & RNA binding motif protein $15 \mathrm{~B}$ & + \\
\hline NM_024834 & 9 & $7.30 \times 10^{-9}$ & C10orf119 & Chromosome 10 open reading frame 119 & + \\
\hline AA196770 & 7 & $1.52 \times 10^{-4}$ & $A L S 2 C R 4$ & Amyotrophic lateral sclerosis 2 (juvenile) chromosome region, ca & + \\
\hline U31383 & 7 & $1.58 \times 10^{-4}$ & GNG10 & Guanine nucleotide binding protein (G protein), $\gamma 10$ & + \\
\hline NM_173060 & 7 & $1.63 \times 10^{-4}$ & CAST & Calpastatin & - \\
\hline AI039422 & 7 & $2.29 \times 10^{-4}$ & RAVER2 & Ribonucleoprotein, PTB-binding 2 & + \\
\hline T86339 & 6 & $3.38 \times 10^{-5}$ & $T N C$ & Tenascin C (hexabrachion) & + \\
\hline AA609551 & 6 & $1.31 \times 10^{-4}$ & & Transcribed locus & + \\
\hline ВC075836 & 6 & $4.91 \times 10^{-4}$ & $R B B P 4$ & Retinoblastoma binding protein 4 & + \\
\hline NM_016097 & 4 & $5.74 \times 10^{-4}$ & IER3IP1 & Immediate early response 3 interacting protein 1 & + \\
\hline ВC015936 & 4 & $6.20 \times 10^{-4}$ & ASH $2 L$ & Ash2 (absent, small, or homeotic)-like (Drosophila) & + \\
\hline NM_021727 & 4 & $6.51 \times 10^{-4}$ & $F A D S 3$ & Fatty acid desaturase 3 & - \\
\hline NM_002733 & 4 & $6.55 \times 10^{-4}$ & PRKAG1 & Protein kinase, AMP-activated, $\gamma 1$ non-catalytic subunit & - \\
\hline
\end{tabular}

${ }^{a}$ Number of selected times as significantly discriminating genes in ten different random permutation tests. ${ }^{\mathrm{b} P}$-values were calculated by random permutation tests with cases with continuous response (group $\mathrm{A}, \mathrm{n}=8$ ) and without (group $\mathrm{B}, \mathrm{n}=11$ ). ${ }^{\mathrm{c}}$ Plus indicates the genes upregulated in group A and minus indicates the genes up-regulated in group B. Information was retrieved from Unigene database in National Center for Biotechnology information.

A)

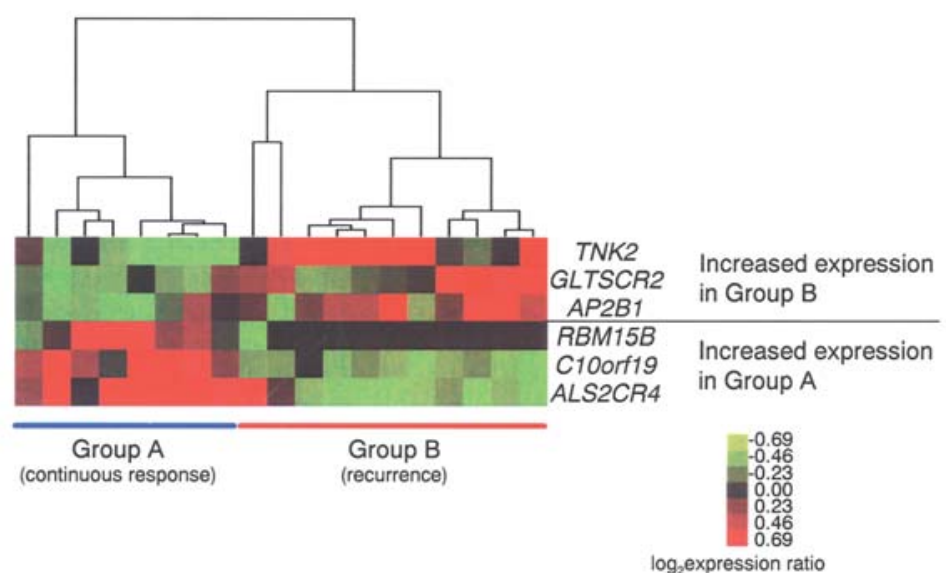

Figure 2. Clustering analysis of 6 predictive genes and distribution of prediction scores for 26 patients. (A) Hierarchical clustering of 19 'learning' cases using 6 predictive genes for recurrence. The dendrograms represent similarities in expression patterns among individual cases; longer branches indicate greater differences. All samples fell appropriately into one of two trees according to their response to imatinib-combined chemotherapy. Three genes were upregulated in patients with continuous response, while the other three genes were up-regulated in patients without continuous response. (B) The prediction score for individual patients using the 6 discriminating genes. The symbol for white circles (cases with continuous response) and black circles (cases without continuous response) indicate scores in cross validation test of patients whose expression data were used for selecting discriminating genes (learning). The symbols for white squares represent scores for four test cases with continuous response, and black squares represent scores for three cases without sufficient decreases of $B C R$ - $A b l$ levels after induction therapy. High absolute values show high confidence. 
Table IV. Correlation of microarray expression data with quantitative-PCR derived values.

\begin{tabular}{llclcl}
\hline Accession no. & Symbol & Pearson correlation coefficient & P-value & Spearman rank correlation & P-value \\
\hline BC044310 & TNK2 & 0.84 & 0.001 & 0.90 & 0.00006 \\
NM_015710 & GLTSCR2 & 0.73 & 0.003 & 0.70 & 0.006 \\
NM_001282 & AP2B1 & 0.65 & 0.002 & 0.50 & 0.029 \\
NM_013286 & RBM15B & 0.72 & 0.03 & 0.80 & 0.01 \\
NM_024834 & C10orf119 & 0.66 & 0.003 & 0.72 & 0.001 \\
AA196770 & ALS2CR4 & 0.82 & 0.00003 & 0.84 & 0.00001
\end{tabular}

A)
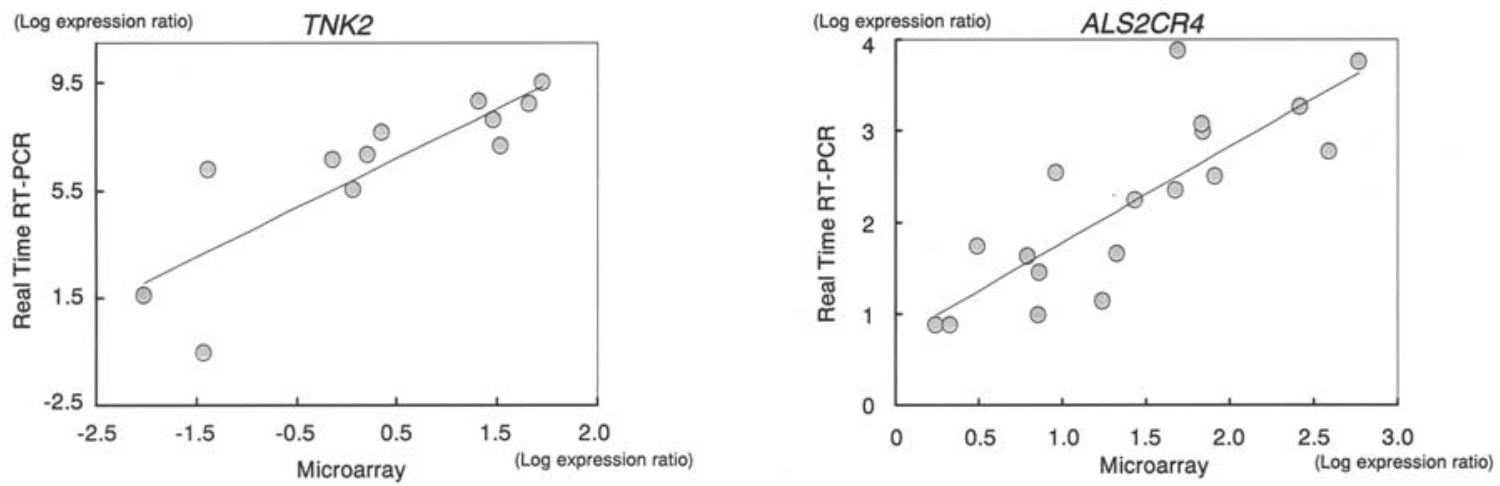

B)
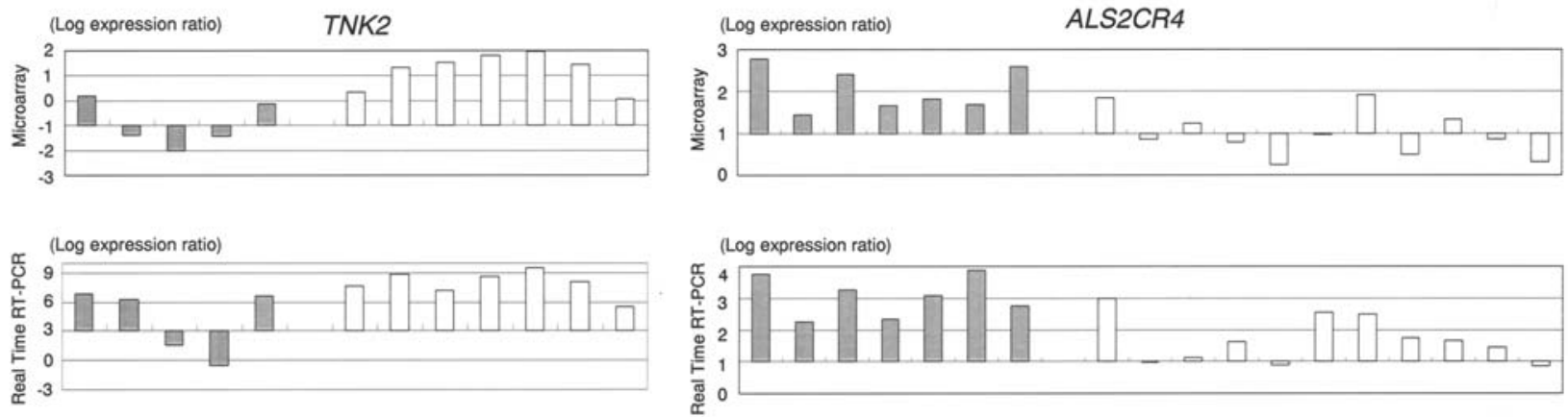

Figure 3. Comparison of microarray-expression data for two genes with quantitative RT-PCR data. (A) Correlation between microarray data for TNK2 (left) and ALS2CR4 (right) and values derived from quantitative RT-PCR experiments. Pearson correlation coefficients of TNK2 and ALS2CR4 were 0.84 (p=0.001) and 0.82 ( $\mathrm{p}=0.00003$ ) respectively. (B) Microarray (above) and quantitative RT-PCR (below) data for TNK2 (left) and ALS2CR4 (right). Black bars in the histogram represent expression of cases with continuous response; white bars represent expression of cases without continuous response.

We calculated the classification score (CS) using the prediction scores of eight group A and eleven group B samples in each gene set, and obtained the best separation of the two groups by using the top 6 genes in our candidategene list (Fig. 2 and Table III). A supervised hierarchical clustering analysis using this set of genes with Cluster and Treeview software (http://rana.lbl.gov/EisenSoftware.htm) yielded good separation of the two groups with regard to absence or presence of the recurrence within 4 months after CR (Fig. 2A).

Finally, to verify the prediction scoring system based on expression data for this set of 6 genes, we examined 4 'test' cases (Fig. 2B). We investigated gene expression profiles in each of the four test cases and then calculated a prediction score for each sample. As shown in Fig. 2B, all of the four cases in group A revealed positive scores indicating the prediction scores for the 4 test cases correctly reflected their clinical response although the number of the cases is too small to make any solid conclusion. Our data suggest that expression levels of these six genes or a part of them might play some roles in the molecular mechanism related to the disease recurrence after achievement of CR by the imatinibcombined chemotherapy. Moreover, we investigated gene expression profiles in each of three cases whose BCR-Abl transcript levels failed to reduce sufficiently after induction therapy (group C). Prediction scores for these three samples calculated from the expression levels of the six genes were negative as for the cases in group B (Fig. 2B). 


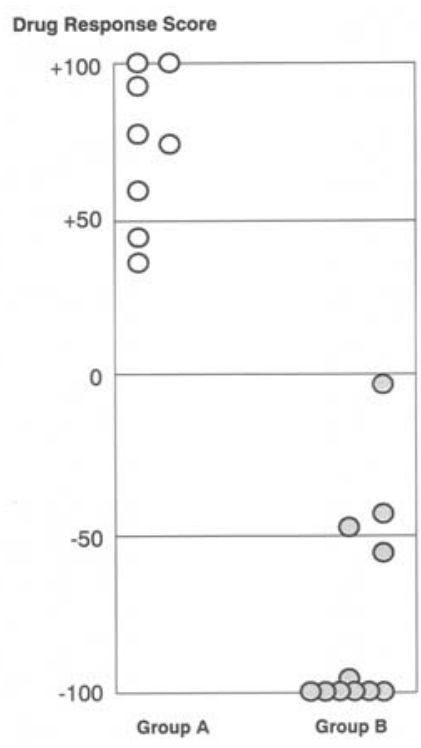

Figure 4. Quantitative reverse transcription-PCR-based prediction scoring system. Prediction scores for 19 cases using values derived from quantitative RT-PCR experiments with 6 predictive genes. White circles indicate scores for cases with continuous response (group A). Black circles indicate scores for cases without continuous response (group B)

Validation of expression measurements. To further validate the results of cDNA microarray analysis, we carried out real-time quantitative RT-PCR for the 6 predictive genes and four quantitative-control genes, PGK1, ACTB, CCT6A and SFRS4, using 19 original (learning) cases. We observed significant concordance between the results from the cDNA microarray and those of the quantitative RT-PCR experiments. As shown in Table IV, Pearson and Spearman rank correlation were positive and statistically significant for all of them. In particular, TNK2 (tyrosine kinase, non-receptor, 2) was significantly more highly expressed in group B than in group A (Fig. 3). On the other hand, expression of ALS2CR4 [amyotrophic lateral sclerosis 2 (juvenile) chromosome region, candidate 4] was significantly lower in group B than in group A (Fig. 3). The quantitative RT-PCR data were significantly concordant with microarray expression data for both genes (TNK2: $\mathrm{r}=0.84, \mathrm{P}<0.001 ; A L S 2$ CR 4: $\mathrm{r}=0.82, \mathrm{P}<0.00003$; Table IV).

Establishment of a quantitative reverse transcription-PCRbased predictive scoring system. To examine the possibility of adapting our prediction system for clinical use, we attempted to establish a scoring system based on quantitative RT-PCR results. We performed quantitative real-time RTPCR of the 6 predictive genes for 19 learning cases ( 8 in group A and 11 in group B), and calculated the prediction score for each case. When we estimated these scores by the leave-one-out cross validation test, all cases were placed correctly according to their response to imatinib-combined chemotherapy (Fig. 4).

\section{Discussion}

Despite relatively high CR rates achieved by standard induction chemotherapies, nearly all patients with $\mathrm{Ph}+\mathrm{ALL}$ experience relapse within a short period of time and die of the disease (9-11). The phase II clinical trial testing imatinibcombined chemotherapy demonstrated that remission induction therapy resulted in CR in 77 (96.2\%) of 80 patients, but early relapse was observed in 20 cases (27). Imatinibcombined regimen appeared effective and feasible, but in some cases, treatment response does not last for a long period. Hence, patients who are at higher risk for relapse may benefit from receiving allogeneic HSCT soon after achievement of CR. However, at the same time, it should be noted that 13 among the 49 patients who received allogeneic HSCT died of transplantation-related complications. Although allogeneic HSCT is a curative form of treatment available for adults with this disease, the procedure itself can be a major cause of treatment failure. Additionally, development of novel tyrosine kinase inhibitors such as dasatinib and nilotinib will further expand the treatment options for this disease $(28,29)$. Under these circumstances, we believe that prediction for response to imatinib-combined chemotherapy should be of great use in clinical decision making.

For such purpose, we analyzed the gene expression profile of $\mathrm{Ph}+\mathrm{ALL}$ cells using cDNA microarray system consisting of 27,648 genes and attempted to establish the prediction system for risk of disease recurrence in patients treated with imatinib-combined chemotherapy. Although other groups have reported the relation between resistance of Ph+ALL to STI571 and gene-expression profiles, this report is the first that shows an ability to predict the response to imatinib-combined therapy by gene expression profiles (35).

By statistical analysis of gene-expression profiles of ALL samples, we identified 16 genes whose expression levels might be associated with the risk of recurrence. The list of 16 genes that showed significant differences between the two groups might provide insight into the biological mechanism(s) involved with treatment response. Among them, the top 6 genes we used for calculation of the prediction score, included tyrosine kinase, non-receptor, 2 (TNK2) and adaptor-related protein complex $2, \beta 1$ subunit $(A P 2 B 1)$ that were up-regulated in group B (patients without continuous response). TNK2 is a tyrosine kinase that inhibits both its intrinsic and stimulated GTPase activity after binding to CDC42 (36-38). On the other hand, AP2B1 is a component of the adaptor complexes which link clathrin to receptors in coated vesicles (36-38). The interaction of TNK2 in vivo with clathrin and AP-2 participated in various trafficking pathways, underlying an ability to increase receptor-mediated transferrin uptake $(37,39)$. It is well known that iron is essential for cell proliferation, and the iron was suggested to enhance the cell proliferation or anti-apoptotic effect (40-43). The up-regulation of TNK2 and $A P 2 B 1$ might induce the iron uptake into the leukemic cells, and the iron might play an important role for re-growth of the leukemic cells.

The interaction between integrin $\beta 1$, which is a subunit of very late antigen (VLA4), on leukemia cells and fibronectin on stromal cells in bone marrow is thought to be crucial in the development of minimal residual disease (MRD) which causes leukemia relapse after chemotherapy (44-47). The up-regulation of calpastatin which inactivates the calpain might inhibit the integrin-mediated adhesion 
disassembly and play an important role in the development of MRD. On the other hand, calpastatin (CAST) that was observed to be up-regulated in group B (Table III) is a specific inhibitor of calpain (calcium-dependent cysteine protease) that is involved in several key aspects of migration through the integrin-mediated signaling (48-50).

Using cDNA microarray, we established a prediction scoring system on the basis of expression levels of the mostsignificantly-associated 6 genes (sub-selected from the 16 genes) that distinguished clearly patients with continuous response from those without. The prediction scoring system using the 6 genes also correctly indicated all of four 'test' cases with continuous response to imatinib-combined chemotherapy although validation of the system using a larger patient size is warranted. Moreover, three cases with insufficient decreases of BCR-Abl transcript levels after induction therapy (group C) were classified into group B (patients without continuous response) category by this prediction system. In any case, it should also be noted that the functions of 4 of our 6 predictive genes are still not well known. Therefore, further investigations will be needed to clarify their biological mechanisms associated with treatment outcome.

Recently other groups have predicted prognosis or chemosensitivity of tumors based on quantitative RT-PCR results for expression of genes selected on microarrays (51). To confirm the reliability of microarray data and investigate a possibility of more convenient prediction strategies in the routine clinical use, we also performed quantitative RT-PCR experiments using the 6 predictive genes for 19 learning cases. We confirmed statistically significant correlation between the data obtained from cDNA microarray and those of quantitative RT-PCR (Table IV; Fig. 3). Moreover, we verified that our quantitative RT-PCR-based prediction system could also correctly classify all of the 19 learning cases with regard to their response to treatment (Fig. 4). Although separation of the two groups by quantitative RTPCR using a set of 6 genes was not so clear as that done by the cDNA microarray analysis, the results are still convincing to separate the two groups.

In conclusion, we are confident that the system based on microarray-derived expression profiles or quantitative RTPCR would provide a good prediction for disease recurrence for patients with $\mathrm{Ph}+\mathrm{ALL}$ treated with imatinib-combined chemotherapy and it would bring significant clinical benefits, although a larger scale study is certainly warranted. Our data suggest that the goal of 'personalized medicine' prescribing the correct treatment to each patient with $\mathrm{Ph}+\mathrm{ALL}$, may be achievable by selecting specific sets of genes by the approach shown here.

\section{Acknowledgments}

We thank Risa Goswammi for cDNA microarray experiments; Noriko Sudo and Miwako Ando for fabricating the cDNA microarray; Drs Tatsuhiko Tsunoda, Fuyuki Miya, Takashi Morizono for analysis of data; and Drs Akihiro Tomita and Ryo Takata for helpful discussions. We also thank all physicians at the JALSG referring centers for providing patient samples, and Dr Masayuki Towatari for his outstanding contribution to the launching of this study. This work was supported in part by the Grant for Cancer Translational Research Project from Ministry of Education, Culture, Sports, Science, and Technology, Government of Japan.

\section{References}

1. Faderl S, Kantarjian HM, Talpaz M and Estrov Z: Clinical significance of minimal residual disease in leukemia. Int $\mathrm{J}$ Oncol 17: 1277-1287, 2000.

2. Schlieben S, Borkhardt A, Reinisch I, et al: Incidence and clinical outcome of children with BCR/ABL-positive acute lymphoblastic leukemia (ALL). A prospective RT-PCR study based on 673 patients enrolled in the German pediatric multicenter therapy trials ALL-BFM-90 and CoALL-05-92. Leukemia 10: 957-963, 1996.

3. Gokbuget N, Hoelzer D, Arnold R, et al: Treatment of Adult ALL according to protocols of the German Multicenter Study Group for Adult ALL (GMALL). Hematol Oncol Clin North Am 14: 1307-1325, 2000.

4. Garcia-Manero G and Kantarjian HM: The hyper-CVAD regimen in adult acute lymphocytic leukemia. Hematol Oncol Clin North Am 14: 1381-1396, 2000.

5. Preti HA, O'Brien S, Giralt S, Beran M, Pierce S and Kantarjian HM: Philadelphia-chromosome-positive adult acute lymphocytic leukemia: characteristics, treatment results, and prognosis in 41 patients. Am J Med 97: 60-65, 1994.

6. Thiebaut A, Vernant JP, Degos L, et al: Adult acute lymphocytic leukemia study testing chemotherapy and autologous and allogeneic transplantation. A follow-up report of the French protocol LALA 87. Hematol Oncol Clin North Am 14: 1353-1366, 2000.

7. Larson RA: Recent clinical trials in acute lymphocytic leukemia by the Cancer and Leukemia group B. Hematol Oncol Clin North Am 14: 1367-1379, 2000.

8. Arico M, Valsecchi MG, Camitta B, et al: Outcome of treatment in children with Philadelphia chromosome-positive acute lymphoblastic leukemia. N Engl J Med 342: 998-1006, 2000.

9. Forman SJ, O'Donnell MR, Nademanee AP, et al: Bone marrow transplantation for patients with Philadelphia chromosomepositive acute lymphoblastic leukemia. Blood 70: 587-588, 1987.

10. Radich JP: Philadelphia chromosome-positive acute lymphocytic leukemia. Hematol Oncol Clin North Am 15: 21-36, 2001.

11. Schrappe M, Arico M, Harbott J, et al: Philadelphia chromosome-positive $(\mathrm{Ph}+)$ childhood acute lymphoblastic leukemia: good initial steroid response allows early prediction of a favorable treatment outcome. Blood 92: 2730-2741, 1998.

12. Snyder DS: Allogeneic stem cell transplantation for Philadelphia chromosome-positive acute lymphoblastic leukemia. Biol Blood Marrow Transplant 6: 597-603, 2000.

13. Avivi I and Goldstone AH: Bone marrow transplant in $\mathrm{Ph}+$ ALL patients. Bone Marrow Transplant 31: 623-632, 2003.

14. Barrett AJ, Horowitz MM, Ash RC, et al: Bone marrow transplantation for Philadelphia chromosome-positive acute lymphoblastic leukemia. Blood 79: 3067-3070, 1992.

15. Schindler T, Bornmann W, Pellicena P, Miller WT, Clarkson B and Kuriyan J: Structural mechanism for STI-571 inhibition of abelson tyrosine kinase. Science 289: 1938-1942, 2000.

16. Mauro MJ, O'Dwyer M, Heinrich MC and Druker BJ: STI571: a paradigm of new agents for cancer therapeutics. J Clin Oncol 20: 325-334, 2002.

17. Kantarjian H, Sawyers C, Hochhaus A, et al: Hematologic and cytogenetic responses to imatinib mesylate in chronic myelogenous leukemia. N Engl J Med 346: 645-652, 2002.

18. Ottmann OG, Druker BJ, Sawyers CL, et al: A phase 2 study of imatinib in patients with relapsed or refractory Philadelphia chromosome-positive acute lymphoid leukemias. Blood 100: 1965-1971, 2002.

19. Hochhaus A, Kreil S, Corbin AS, et al: Molecular and chromosomal mechanisms of resistance to imatinib (STI571) therapy. Leukemia 16: 2190-2196, 2002.

20. Thomas DA, Faderl S, Cortes J, et al: Treatment of Philadelphia chromosome-positive acute lymphocytic leukemia with hyperCVAD and imatinib mesylate. Blood 103: 4396-4407, 2004.

21. Von Bubnoff N, Peschel C and Duyster J: Resistance of Philadelphia-chromosome positive leukemia towards the kinase inhibitor imatinib (STI571, Glivec): a targeted oncoprotein strikes back. Leukemia 17: 829-838, 2003. 
22. O'Brien SG, Guilhot F, Larson RA, et al: Imatinib compared with interferon and low-dose cytarabine for newly diagnosed chronic-phase chronic myeloid leukemia. N Engl J Med 348: 994-1004, 2003.

23. Druker BJ, Sawyers CL, Kantarjian H, et al: Activity of a specific inhibitor of the BCR-ABL tyrosine kinase in the blast crisis of chronic myeloid leukemia and acute lymphoblastic leukemia with the Philadelphia chromosome. N Engl J Med 344: 1038-1042, 2001

24. De Labarthe A, Rousselot P, Huguet-Rigal F, et al: Imatinib combined with induction or consolidation chemotherapy in patients with de novo Philadelphia chromosome-positive acute lymphoblastic leukemia - results of the GRAAPH-2003 study. Blood 27: 22, 2006.

25. Towatari M, Yanada M, Usui N, et al: Combination of intensive chemotherapy and imatinib can rapidly induce high-quality complete remission for a majority of patients with newly diagnosed BCR-ABL-positive acute lymphoblastic leukemia. Blood 104: 3507-3512, 2004.

26. Wassmann B, Pfeifer H, Goekbuget N, et al: Alternating versus concurrent schedules of imatinib and chemotherapy as front-line therapy for Philadelphia-positive acute lymphoblastic leukemia (Ph+ALL). Blood 108: 1469-1477, 2006.

27. Yanada M, Takeuchi J, Sugiura I, et al: High complete remission rate and promising outcome by combination of imatinib and chemotherapy for newly diagnosed BCR-ABL-positive acute lymphoblastic leukemia: a phase II study by the Japan Adult Leukemia Study Group. J Clin Oncol 24: 460-466, 2006.

28. Talpaz M, Shah NP, Kantarjian H, et al: Dasatinib in imatinibresistant Philadelphia chromosome-positive leukemias. N Engl J Med 354: 2531-2541, 2006.

29. Kantarjian H, Giles F, Wunderle L, et al: Nilotinib in imatinibresistant CML and Philadelphia chromosome-positive ALL. N Engl J Med 354: 2542-2551, 2006.

30. Ono K, Tanaka T, Tsunoda T, et al: Identification by cDNA microarray of genes involved in ovarian carcinogenesis. Cancer Res 60: 5007-5011, 2000.

31. Okabe H, Satoh S, Kato T, et al: Genome-wide analysis of gene expression in human hepatocellular carcinomas using cDNA microarray: identification of genes involved in viral carcinogenesis and tumor progression. Cancer Res 61: 2129-2137, 2001 .

32. Zembutsu H, Ohnishi Y, Tsunoda T, et al: Genome-wide cDNA microarray screening to correlate gene expression profiles with sensitivity of 85 human cancer xenografts to anticancer drugs. Cancer Res 62: 518-527, 2002.

33. Golub TR, Slonim DK, Tamayo $\mathrm{P}$, et al: Molecular classification of cancer: class discovery and class prediction by gene expression monitoring. Science 286: 531-537, 1999.

34. Takata R, Katagiri T, Kanehira M, et al: Predicting response to methotrexate, vinblastine, doxorubicin, and cisplatin neoadjuvant chemotherapy for bladder cancers through genomewide gene expression profiling. Clin Cancer Res 11:2625-2636, 2005.

35. Hofmann WK, De Vos S, Elashoff D, et al: Relation between resistance of Philadelphia-chromosome-positive acute lymphoblastic leukaemia to the tyrosine kinase inhibitor STI571 and gene-expression profiles: a gene-expression study. Lancet 359: 481-486, 2002.
36. Yang W, Lo CG, Dispenza T and Cerione RA: The Cdc42 target ACK2 directly interacts with clathrin and influences clathrin assembly. J Biol Chem 276: 17468-17473, 2001.

37. Teo M, Tan L, Lim L and Manser E: The tyrosine kinase ACK1 associates with clathrin-coated vesicles through a binding motif shared by arrestin and other adaptors. J Biol Chem 276 : 18392-18398, 2001.

38. Ahmed I, Calle Y, Sayed MA, et al: Cdc42-dependent nuclear translocation of non-receptor tyrosine kinase, ACK. Biochem Biophys Res Commun 314: 571-579, 2004.

39. Meyerholz A, Hinrichsen L, Groos S, Esk PC, Brandes G and Ungewickell EJ: Effect of clathrin assembly lymphoid myeloid leukemia protein depletion on clathrin coat formation. Traffic 6 : 1225-1234, 2005.

40. Kovar J, Valenta T and Stybrova H: Differing sensitivity of tumor cells to apoptosis induced by iron deprivation in vitro. In Vitro Cell Dev Biol Anim 37: 450-458, 2001.

41. Leardi A, Caraglia M, Selleri C, et al: Desferioxamine increases iron depletion and apoptosis induced by ara-C of human myeloid leukaemic cells. Br J Haematol 102: 746-752, 1998.

42. Potaznik D, Groshen S, Miller D, et al: Association of serum iron, serum transferrin saturation, and serum ferritin with survival in acute lymphocytic leukemia. Am J Pediatr Hematol Oncol 9: 350-355, 1987.

43. Moura IC, Lepelletier Y, Arnulf B, et al: A neutralizing monoclonal antibody (mAb A24) directed against the transferrin receptor induces apoptosis of tumor T lymphocytes from ATL patients. Blood 103: 1838-1845, 2004.

44. Hutchinson E: Clinging on. Nat Rev Cancer 3: 717, 2003.

45. Irving JA, Minto L, Bailey S and Hall AG: Loss of heterozygosity and somatic mutations of the glucocorticoid receptor gene are rarely found at relapse in pediatric acute lymphoblastic leukemia but may occur in a subpopulation early in the disease course. Cancer Res 65: 9712-9718, 2005.

46. Kirschner-Schwabe R, Lottaz C, Todling J, et al: Expression of late cell cycle genes and an increased proliferative capacity characterize very early relapse of childhood acute lymphoblastic leukemia. Clin Cancer Res 12: 4553-4561, 2006.

47. Matsunaga T, Takemoto N, Sato T, et al: Interaction between leukemic-cell VLA-4 and stromal fibronectin is a decisive factor for minimal residual disease of acute myelogenous leukemia. Nat Med 9: 1158-1165, 2003.

48. Franco SJ and Huttenlocher A: Regulating cell migration: calpains make the cut. J Cell Sci 118: 3829-3838, 2005.

49. Yuan Y, Dopheide SM, Ivanidis C, Salem HH and Jackson SP: Calpain regulation of cytoskeletal signaling complexes in von Willebrand factor-stimulated platelets. Distinct roles for glycoprotein Ib-V-IX and glycoprotein IIb-IIIa (integrin alphaIlbbeta3) in von Willebrand factor-induced signal transduction. J Biol Chem 272: 21847-21854, 1997.

50. Yan B, Calderwood DA, Yaspan B and Ginsberg MH: Calpain cleavage promotes talin binding to the beta 3 integrin cytoplasmic domain. J Biol Chem 276: 28164-28170, 2001.

51. Ma XJ, Wang Z, Ryan PD, et al: A two-gene expression ratio predicts clinical outcome in breast cancer patients treated with tamoxifen. Cancer Cell 5: 607-616, 2004. 